\title{
Intermittent Loss of Power
}

National Cancer Institute

\section{Source}

National Cancer Institute. Intermittent Loss of Power. NCI Thesaurus. Code C133520.

Problem associated with an intermittent disruption to the power to run the device. 